\title{
Xanthoma disseminatum with respiratory tract involvement and fatal outcome
}

\author{
Christopher W H Davies, Pauline Marren, Mark C Juniper, Winifred Gray, \\ Fenella Wojnorowska, Malcolm K Benson
}

\begin{abstract}
Xanthoma disseminatum (XD) is a rare mucocutaneous xanthomatosis classified as a benign form of non-Langerhans' cell histiocytosis. The case history is presented of a 61 year old woman with XD who developed dyspnoea and spirometric features of airflow obstruction. Bronchoscopy and computed tomography confirmed involvement of the large and medium sized bronchi and she subsequently died from acute respiratory failure. The post-mortem findings and the importance of respiratory tract disease in this unusual condition are discussed. (Thorax 2000;55:170-172)
\end{abstract}

Keywords: xanthoma disseminatum; respiratory tract

Xanthoma disseminatum (XD) is a rare, benign mucocutaneous xanthomatosis caused by the proliferation of non-X histiocytic cells. It is characterised by widespread cutaneous xanthomas ${ }^{12}$ and may affect the mucous membranes of the mouth and upper respiratory tract, but involvement of the lower respiratory tract is extremely uncommon. We present a case of XD affecting the skin, upper airways, and also the lower respiratory tract leading to death by respiratory failure. The importance of respiratory tract involvement in XD is discussed.

Osler Chest Unit,

Churchill Hospital, Oxford Radcliffe

Trust, Oxford OX3

7LJ, UK

C W H Davies

M C Juniper

M K Benson

\section{Department of}

Dermatology

P Marren

F Wojnorowska

Department of Cellular Pathology,

John Radcliffe

Hospital, Oxford

Radcliffe Trust, Oxford

OX3 9DU, UK

W Gray

Correspondence to:

Dr C W H Davies

Received 22 August 1997

Returned to authors

2 February 1998

Revised manuscript received

16 April 1998

Accepted for publication

1 May 1998 shaped histiocytes in the larger nodular lesions. Immunocytochemical tests were negative for markers of histiocytosis-X (S-100 protein, LN3 and peanut agglutination) and positive for factor XIIIa, HAM 56, and KP1. There were no cells containing Birbeck granules at electron microscopy and the cells showed an irregular scalloped border with a centrally placed nucleus. In view of the clinicopathological presentation and immunocytochemical markers the diagnosis of xanthoma disseminatum was confirmed. She was commenced on topical clobetasol propionate $0.05 \%$; over the following few months she developed lesions elsewhere on the trunk and buttocks with little response to the topical steroids.

In February 1995 she developed dyspnoea, wheeze, and a weak voice. Examination at that time revealed a fixed wheeze in the left lung and slight hoarseness of the voice. Spirometric tests revealed obstructive airflow with a forced expiratory volume in one second $\left(\mathrm{FEV}_{1}\right)$ of 1.0 litre $(40 \%$ predicted) and forced vital capacity (FVC) of 2.5 litres ( $85 \%$ predicted). The chest radiograph was unremarkable. Bronchoscopic examination showed bilateral vocal cord oedema and a nodule at the anterior commissure. Throughout the trachea and main airways there were numerous flat pale white lesions and biopsy specimens showed a mucosal infiltrate of histiocytic cells with a foamy cytoplasm. There was no evidence of granuloma formation and Ziell-Nielsen stain failed to reveal acid fast bacilli. Blood investigations including serum angiotensin converting enzyme (ACE) were normal. She was commenced on oral prednisolone $10 \mathrm{mg}$ daily and after six months of monitored treatment there had been no subjective or objective evidence of improvement in the chest. The prednisolone was reduced to $5 \mathrm{mg}$ daily without deterioration in symptoms.

In 1996 she was reviewed and still complained of a weak voice and dyspnoea. During the interval she had continued to develop further cutaneous lesions consistent with XD. Chest examination was normal. Pulmonary function tests were unchanged and confirmed airflow obstruction: $\mathrm{FEV}_{1} 1.0$ litre (40\% predicted) and FVC 2.6 litres (87\% predicted) with normal gas transfer. Flow volume loops excluded fixed upper airway obstruction. Computed tomographic (CT) scanning of the thorax showed significant thickening of the trachea and large airways, and minor bronchial wall thickening throughout the lungs. There was no evidence of interstitial lung disease. Blood investigations were again normal. She was given a trial of inhaled beclomethasone which had no beneficial effect and was discontinued after two months. 


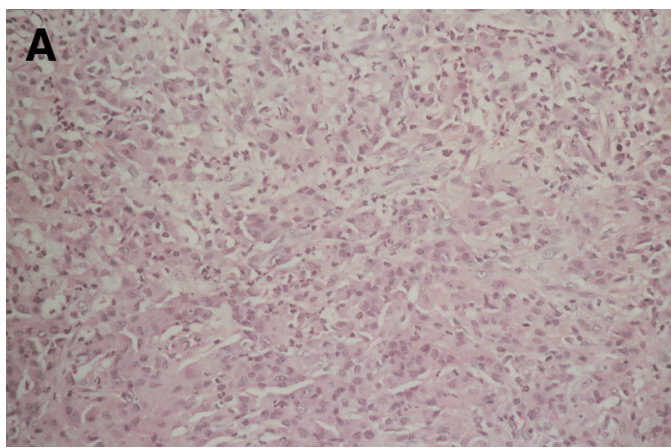

B
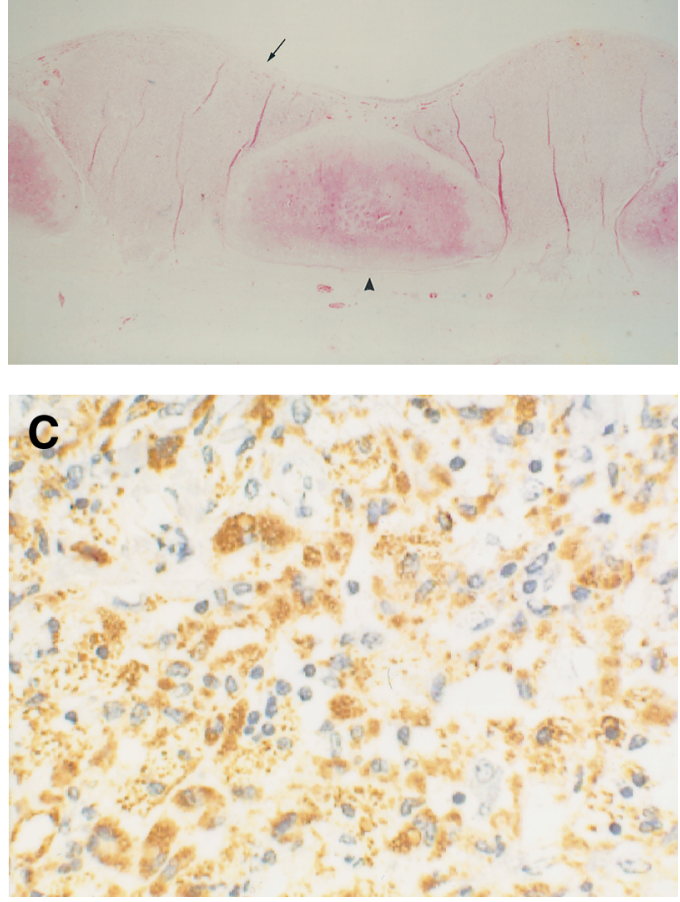

Figure 1 (A) Section of skin showing mixed cellular infiltrate composed of pale vacuolated histiocytic cells with some multinucleated forms. Lymphocytes and other inflammatory cells are present in the background (stain: $H$ $\mathcal{E} E$; high power magnification). (B) Section of trachea (post mortem) showing nodular masses of pale tissue (arrow) consisting almost entirely of histiocytes with a few giant cells. The nodules are interspersed between the cartilage plates (arrow head) (stain: $H \mathcal{E} E$; medium power magnification). (C) Immunostaining of section of trachea using KP1 showing positive staining of macrophages (high power magnification).

The patient was reassessed in 1997 because of worsening dyspnoea. Chest examination was again unremarkable and oxygen saturation was $95 \%$. FEV had fallen slightly to 0.8 litres. A repeat bronchoscopic examination showed small submucosal nodules in the trachea and main airways, but biopsy specimens were nondiagnostic.

Forty eight hours later she became increasingly unwell and suffered a respiratory arrest at home. Resuscitation was unsuccessful and necroscopic examination confirmed numerous submucosal nodules in the epiglottis, trachea, and all the bronchi (fig 1B). Nodules were also present on the visceral pleura, the epicardium, and the lower third of the oesophagus. Histologically all of these tissues showed infiltration of foamy macrophages and histiocytic proliferation with lipid storing cells and immunocytochemical examination confirmed that they were also non-X histiocytic cells (fig 1C). There was no cytological atypia or evidence of neoplastic transformation in any of the sites; these have been compared with histological examination of the skin and confirm the appearances of XD. The cause of death was stated as respiratory failure and bronchospasm secondary to XD.

\section{Discussion}

Xanthoma disseminatum is one of several heterogeneous conditions known collectively as the primary histiocytic dermatose ${ }^{12}$ or cutaneous syndromes of non-X histiocytoses, ${ }^{3}$ so called to differentiate them from the more recognised condition histiocytosis-X (HX). The condition is caused by a proliferation of non-X histiocytic cells, probably from a macrophage/ monocyte origin, ${ }^{4}$ and was probably first described in 1867 by von $\mathrm{Grafe}^{5}$ and subsequently as a distinct entity by Montgomery and Osterberg in $1938 .^{6}$ Only about 100 cases of $\mathrm{XD}$ have been reported in the world literature; the condition appears to affect predominantly male children and young adults but has been described in both sexes and in all age groups. ${ }^{7}$

As XD and HX share clinical and pathological features, difficulties may arise in diagnosis but immunocytochemical and ultrastructural differences have recently been recognised. ${ }^{4}$ Electron microscopy shows Birbeck (Langerhans') granules in $\mathrm{HX}$ which are absent in XD, while more phagosomes are seen in $\mathrm{XD}$ and other non-X histiocytoses compared with $\mathrm{HX} .{ }^{49}$

Immunocytochemistry has shown differences between the proliferating Langerhans' cells in HX which label positively for S-100, LN3, and peanut agglutination in contrast to cells of macrophage/monocyte origin in XD (and other non-X histiocytoses) which are strongly positive for factor XIIIa, KiM6, and KP1 markers. ${ }^{4} 9$

Typically XD affects the flexural areas and skin lesions may regress spontaneously after several years. ${ }^{189}$ More commonly a persistent mucocutaneous form occurs and treatment with steroids, immunosuppressive agents, and radiotherapy is usually unsuccessful ${ }^{128-10} \mathrm{de}-$ spite the successful use of agents such as etoposide, the vinca alkaloids, and methylprednisolone in Langerhans' cell histiocytosis. ${ }^{11}$ Selective cutaneous lesions in XD may be ablated by dermabrasion or electrodesiccation. ${ }^{1}$ Diabetes insipidus is a common systemic manifestation of the disease but may be mild and transient ${ }^{2}$ and involvement of mucous membranes may be observed in $30-50 \%$ of cases, typically affecting most frequently (in order) the larynx, pharynx, mouth, trachea, epiglottis, and tongue. ${ }^{2} 89$

When the pharynx and upper airways are involved symptoms of dyspnoea and dysphagia may occur and endoscopic examination may reveal plaques covering the mucosal surfaces. These can cause life threatening obstruction and asphyxiation requiring tracheostomy and has been reported in a total of eight patients. $^{7}$ p12-14 $^{12}$ This is the most recognised cause of morbidity in $\mathrm{XD}$, but tracheostomy 
may successfully relieve obstruction to the larynx with patients continuing in good health at subsequent follow up.

In our patient necroscopy showed no evidence that upper airway obstruction was responsible for asphyxiation but that death was caused by bronchospasm secondary to extensive involvement of the medium and small sized bronchi leading to respiratory failure. This complication has not been described since 1925 when Turner et $a l^{14}$ reported the death of a 22 year old girl with XD who also died from respiratory failure secondary to airway infiltration including the bronchi. Although infiltration of the bronchi has been reported at necropsy in one other case of $\mathrm{XD},{ }^{7}$ it was not the cause of death as seen in our patient.

Xanthoma disseminatum is a rare mucocutaneous xanthomatosis considered to be benign and self-limiting and unresponsive to various modes of treatment. Involvement of the larynx may, however, lead to life threatening complications necessitating tracheostomy with a satisfactory long term prognosis but, when the lower respiratory tract is affected as in this case, the prognosis is poor leading to respiratory failure and death.
1 Ringel E, Moschella S. Primary histiocytic dermatoses. Arch Dermatol 1962;86:582-96.

2 Caputo R. Cutaneous non histiocytoses X. In: Fitzpatrick TB, Eisen AZ, Wolff K, et al, eds. Dermatology in general medicine. Volume 1. 4th ed. New York: McGraw Hill, 1993: 1261-9.

3 Winkelman RK. Cutaneous syndromes of non X histiocytosis. Arch Dermatol 1981;117:667-72.

4 Zelger B, Cerio R, Orchard G, et al. Histologic and immunohistochemical study comparing xanthoma disseminatum and histiocytosis X. Arch Dermatol 1992;128:1207-12.

5 von Gräfe. Verhandlungen ärztlicher Gessellschaften: ein Fall von eigentümlichen Tumoren auf Hornhäuten. Klin Fall von eigentümlich

6 Montgomery H, Osterberg AE. Correlation of clinical, histopathologic and chemical studies of cutaneous xanthomas. Arch Dermatol Syph 1938;37:373-502.

7 Altman J, Winkelman RK. Xanthoma disseminatum. Arch Dermatol 1962;86:582-96.

$8 \mathrm{Chu}$ AC. Histiocytoses. In: Champion RH, Burton JL, Ebling FJG, eds. Textbook of dermatology. Volume 3. 5th ed. Oxford: Blackwell Scientific Publications, 1994: 2041-64

9 Caputo R, Veraldi S, Grimalt R, et al. The various clinical patterns of xanthoma disseminatum. Considerations on seven cases and review of the literature. Dermatology 1995 ; 190:19-24.

10 Mishkel MA, Cockshott WP, Nazir DJ, et al. Xanthoma disseminatum: clinical, metabolic and radiologic aspects. Arch Dermatol 1977;113:1094-100.

11 Ladisch S, Gadner H. Treatment of Langerhans' cell histiocytosis: evolution and current approaches. Brf Cancer Suppl 1994;23:S41-6.

12 Varotti C, Bettoli V, Berti E, et al. Xanthoma disseminatum: a case with extensive mucous membrane involvement. $\mathcal{f}$ Am Acad Dermatol 1991;25:433-6.

13 Kilinc Y, Ozsahinoglu C, Varinli S, et al. An unusual case of xanthoma disseminatum. Int $\mathcal{f}$ Pediatr Otorhinolaryngol 1992;23:73-9.

14 Turner A, Davidson J, White A. Xanthomatosis: some aspects of its blood chemistry and pathology, case 4. Edinburgh Med F 1925;32:153.

\section{LETTERS TO THE EDITOR}

\section{Silica dust and lung cancer}

We appreciate the recent contribution of Ulm et $a l^{1}$ to the controversial question as to whether occupational exposure to crystalline silica, a classified carcinogen, increases the risk of lung cancer irrespective of silicosis. There are, however, some serious conceptua and methodological questions regarding the design, conduct, and analysis of the study which may affect the conclusions.

If silicosis is a surrogate for the internal dose or on the causal pathway between silica exposure and lung cancer, this question might only be addressed with detailed individual exposure information and classification of the silicosis grade in a population of silicotic and non-silicotic subjects.

The authors presented the pooled data from two matched case-control studies among non-silicotic subjects in two German industries. The study populations were not clearly defined with respect to the region. Cohorts for the recruitment of cases and controls as suggested by the authors cannot be reconstructed. Given the lack of a nationwide cancer registry in Germany, complete case ascertainment is questionable. In particular, the selection of potential cases from the workforce due to health problems poses a threat to internal validity.

Recruitment of controls and exposure levels varied between the two industries. Selection of more highly exposed or silicotic control subjects, in particular in the stone and quarrying industry, cannot be ruled out. We think that more detailed information on the data and results from the two industries, which have been presented in a previous German report of this study, ${ }^{2}$ is crucial for the interpretation of the results. Discrepant results between the two industries and between different publications of this study ought to be discussed.

The authors made a great effort to reconstruct the exposure to silica dust. However, the quality and completeness of the exposure data remain questionable because the exposure assessment was retrospective and no figures on missing dust measurements were presented.

The baseline risks of the cases and controls were high (cumulative exposure $\geqslant 0.12 \mathrm{mg}$ / $\mathrm{m}^{3} \cdot$ years) which may render the detection of an additional risk of lung cancer in this study population difficult. Could the restriction to highly exposed individuals explain the low relative risks of lung cancer in this study?

In the previous report of this case-control study, ${ }^{2}$ which included subjects with silicosis, the risk estimates of lung cancer for the three exposure indices were relatively low and not significant. Why do the authors expect the risks and the significance to be higher after excluding those with silicosis?

The authors presented only adjusted odds ratios. We appreciate the control of confounding by other occupational exposures. However, if the duration of exposure is a surrogate for the investigated exposure, an additional risk might not be observed after adjustment for duration of exposure.

The authors matched subjects on smoking status yet, in the previous report, an index of smoking intensity and duration was a significant risk factor for lung cancer and the smoking adjusted risk estimates were slightly higher than the risk estimates reported in this paper. ${ }^{2}$ Why did the authors not adjust for smoking as in the previous report?
In general, details on excluded and deceased subjects, coding of variables, missing data, and statistical methods presented in this paper were not easily verifiable. In the discussion the authors concluded that the study showed no association between exposure to crystalline silica and lung cancer which might either be a true finding or be attributed to a lack of statistical power. Like previous studies of non-silicotic subjects ${ }^{3}$ this study lacked the power to detect risks of 1.4 or less. In addition, all potential sources of bias (nondifferential misclassification of the exposure, selection bias, and healthy worker effect) lead to an underestimation of the risk estimates. We would therefore suggest that the results are interpreted with caution. It remains unclear whether silicosis is on the causal pathway between exposure to silica and lung cancer.

X BAUR

U LATZA

BGFA, Institute at the Rular-Universität Bochum, Bürkle-de-la-Camp-Platz 1, D 44789 Bochum,

Germany

K-H JÖCKEL

Institute for Medical Informatics, Biometry and Epidemiology, University Clinics of Essen, D-45122 Essen, Germany

1 Ulm K, Waschuzik B, Ehnes H, et al. Silicosis and lung cancer in the German stone, quarrying, and ceramics industries: results of case-control study. Thorax 1999;54:347-51.

2 Ulm K, Waschuzik B. Quarzfeinstaub und Lungenkarzinom: Ergebnisse einer FalIkonLungenkarzinom. Ergebnisse einer Fallkontrollstudie zur Untersuchung des Zusammenhangs zwischen einer Exposition gegenübe lungengängigem Quarzfeinstaub und dem Auftreten von Lungenkarzinomen (German). HVBG, St Augustin, 1998.

3 Weill H, McDonald JC. Exposure to crystalline silica and risk of lung cancer: the epidemiological evidence. Thorax 1996;51:97-102.

AUTHORS' REPLY We appreciate the comments of Baur and colleagues on our paper. ${ }^{1}$ In Germany it is difficult to perform epidemiological studies due to the lack of registries and some regulations, therefore in case- 
Table 1 Distribution of the cumulative exposure $\left(\mathrm{mg} / \mathrm{m}^{3} \cdot\right.$ years $)$

\begin{tabular}{llllll}
\hline & \multicolumn{2}{l}{ Stone E quarrying } & & \multicolumn{2}{l}{ Ceramics } \\
\cline { 2 - 3 } \cline { 5 - 5 } & Cases $(n=133)$ & Controls $(n=231)$ & & Cases $(n=114)$ & Controls $(n=564)$ \\
\hline Minimum & 0.12 & 0.12 & 0.15 & 0.18 \\
$10 \%$ & 0.85 & 0.64 & 0.54 & 0.98 \\
$25 \%$ & 1.76 & 1.20 & 1.34 & 1.80 \\
$50 \%$ & 3.24 & 2.61 & 2.70 & 2.91 \\
$75 \%$ & 8.12 & 6.12 & & 4.19 & 6.28 \\
$90 \%$ & 16.13 & 13.10 & 22.32 & 11.52 \\
\hline
\end{tabular}

control studies complete case ascertainment can never be guaranteed. We checked all the sources available to identify all cases with lung cancer in the selected area of Germany. The main question, however, is whether the group of cases enrolled is selected and not a random sample. There are no indications for that bias with respect to the exposure.

The selection of controls can be a problem in case-control studies, especially if there is no file of all possible controls available, which is the situation in the stone and quarrying industry. As already mentioned in the paper, for this type of industry the so-called accident file has been used. The possibility of a selection bias can never be ruled out. However, most of the accidents are based on carelessness such as stumbling. Whether those accidents are related to any exposure levels is highly speculative.

Due to lack of space in the paper the data reported had to be condensed. In table 1 the distribution of the cumulative exposure for both industries are given separately. There is a difference between both industries with respect to higher exposures.

The level of exposure in both industries is comparable to the other studies and not exceptionally high as stated by the authors of the letter. In the monograph by IARC $^{2}$ the geometric mean for the ceramic industry varied between 0.01 and $0.44 \mathrm{mg} / \mathrm{m}^{3}$ with a range of $0.01-1.14 \mathrm{mg} / \mathrm{m}^{3}$. The level in the quarrying industry is higher with a mean value of $0.03-2.1 \mathrm{mg} / \mathrm{m}^{3}$ and peak values over $100 \mathrm{mg} / \mathrm{m}^{3}$.

Assessment of the exposure in occupational epidemiology is mostly based on retrospective estimates and not on measurements. Within this study great effort has been made to quantify the exposure. If measurements were available these have been taken into account.

If the smoking habits are included in the model the fit improves considerably. In the analysis of the average exposure the likelihood ratio value is 386.62 with smoking and 345.72 without. However, the estimates of the odds ratio for the average exposure remain nearly unchanged (with smoking: OR $=0.94, \mathrm{p}=0.82$; without smoking: OR $=$ $0.91 ; \mathrm{p}=0.71$ ).

The exclusion of subjects with silicosis in the final analysis is due to increased knowledge acquired subsequently. After the decision by the IARC in 1996, the main interest was focused on the question of whether or not silicosis is a prerequisite for lung cancer. There are only a few studies available investigating the risk in subjects without silicosis.

Our study is in line with other publications In the absence of silicosis there is either no additional risk caused by silica dust or only a very low risk. For practical purposes it seems justified to check the current threshold values for silica dust in order to prevent silicosis and therefore to exclude, or at least minimise, the associated risk of lung cancer.

K ULM

Institut für Medzinische Statistik und Epidemiologi der Technischen Universität München, Ismaninger Strasse 22, 81675 Munich,

Germany

1 Ulm K, Waschuzik B, Ehnes H, et al. Silicosis and lung cancer in the German stone, quarrying, and ceramics industries: results of a case-control study. Thorax 1999;54:347-51.

2 IARC. Silica, some silicates, coal dust and IARC. Silica, some silicates, coal dust and
para-aramide fibrils. Monographs on the Evalupara-aramide fibrils. Monographs on the Evalu-
ation of Carcinogenic Risks to Humans, Vol 68. Lyon: IARC, 1997.

\section{Re-estimated equations for 5 th percentiles of lung function variables}

In 1996 we published reference equations for the 5 th percentiles of lung function parameters (FVC, FEV, PEF, FEF ${ }_{25}, \mathrm{FEF}_{50}, \mathrm{FEF}_{75}$, $\mathrm{FEF}_{25-75}, \mathrm{FEV}_{1} / \mathrm{FVC}$ ) as a function of age and height. ${ }^{1}$ These reference values were derived from the subsample of the Swiss SAPALDIA study population consisting of never smokers without respiratory symptoms (1267 men and 1890 women aged between 18 and 60 years). In the meantime our German colleagues have applied extrapolations of the 5 th percentile curves to men aged between 60 and 70 years $^{2}$ and have found that for some of the lung function parameters-for example, $\mathrm{FEV}_{1}$ - the 5th percentiles appeared to get too close to the mean at age 70 (fig 1).

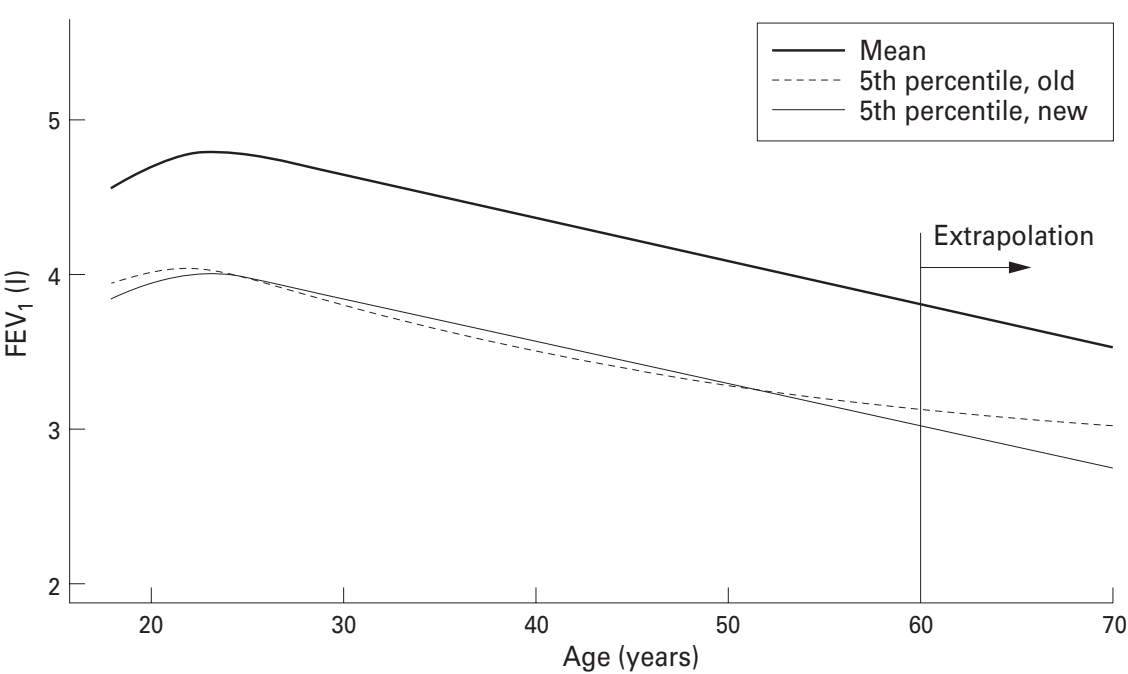

Figure 1 Mean and 5th percentile of FEV in men of height $1.80 \mathrm{~m}$ as a function of age (based on cross sectional lung function data from healthy never smoking men aged 18-60 years). Original and revised estimates of the 5 th percentiles are shown.
A new method of estimating percentile curves provides slightly different estimates of the 5th percentile with more plausible extrapolations. This method uses weighted $\mathrm{L}_{1}$ regression. Thus, instead of minimising the sum of squared residuals, ${ }^{3}$ a weighted sum of the absolute values of the residuals is minimised. For instance, in order to obtain an estimate of the 5 th percentile curve, the weight assigned to terms stemming from negative residuals must be 19 times larger than the weight assigned to terms whose underlying residual is positive. ${ }^{4}$ (As in our original approach, we used the logarithms of individual ratios between observed and predicted lung function values (LF) as the basis for estimating the 5th percentile curves.) These residuals were regressed against age using weighted $\mathrm{L}_{1}$ regression as described above. If $y=\alpha+\beta$ age denotes the estimated regression line for the 5 th percentile of $r=\ln$ (LFobserved/LFpredicted) as a function of age, then $\mathrm{y}=\mathrm{LF}$ predicted $\exp (\alpha$ $+\beta$ age) is an estimate of the 5 th percentile of LF given age. To test whether a linear age term was sufficient to describe the age dependency of the 5th percentile of $r$ we

Table 1 Parameters of the multiplicative factor $f_{0.05}=\exp (\alpha+\beta$ age $)$ by which the predicted value from table 2 must be multiplied in order to get the 5th percentile value for the given age and height

\begin{tabular}{lll}
\hline & $a$ & $\beta$ \\
\hline Men & & \\
FVC & -0.140 & -0.00166 \\
$\mathrm{FEV}_{1}$ & -0.143 & -0.00158 \\
$\mathrm{PEF}$ & -0.313 & -0.00274 \\
$\mathrm{FEF}_{25-75}$ & -0.249 & -0.00676 \\
$\mathrm{FEF}_{25}$ & -0.341 & -0.00269 \\
$\mathrm{FEF}_{50}$ & -0.293 & -0.00491 \\
$\mathrm{FEF}_{75}$ & -0.341 & -0.00758 \\
$\mathrm{FEV}_{1} / \mathrm{FVC}$ & -0.132 & -0.00047 \\
$\mathrm{Women}^{\mathrm{FVC}}$ & & \\
$\mathrm{FEV}_{1}$ & -0.194 & -0.00112 \\
$\mathrm{PEF}$ & -0.174 & -0.00137 \\
$\mathrm{FEF}_{25-75}$ & -0.291 & -0.00349 \\
$\mathrm{FEF}_{25}$ & -0.235 & -0.00599 \\
$\mathrm{FEF}_{50}$ & -0.262 & -0.00402 \\
$\mathrm{FEF}_{75}$ & -0.200 & -0.00638 \\
$\mathrm{FEV}_{1} / \mathrm{FVC}$ & -0.362 & -0.00696 \\
\hline & -0.103 & -0.00059
\end{tabular}

For a man of 20 years and height $180 \mathrm{~cm}$ (for whom, according to table 2, the predicted FEV value is 4.696), the 5th percentile of $\mathrm{FEV}_{1}$ is computed as follows:

$\mathrm{FEV}=4.696 \mathrm{e}^{(-0.143-0.00158 * 20)}=3.944$ 
Table 2 Prediction equations for the means of lung function variables

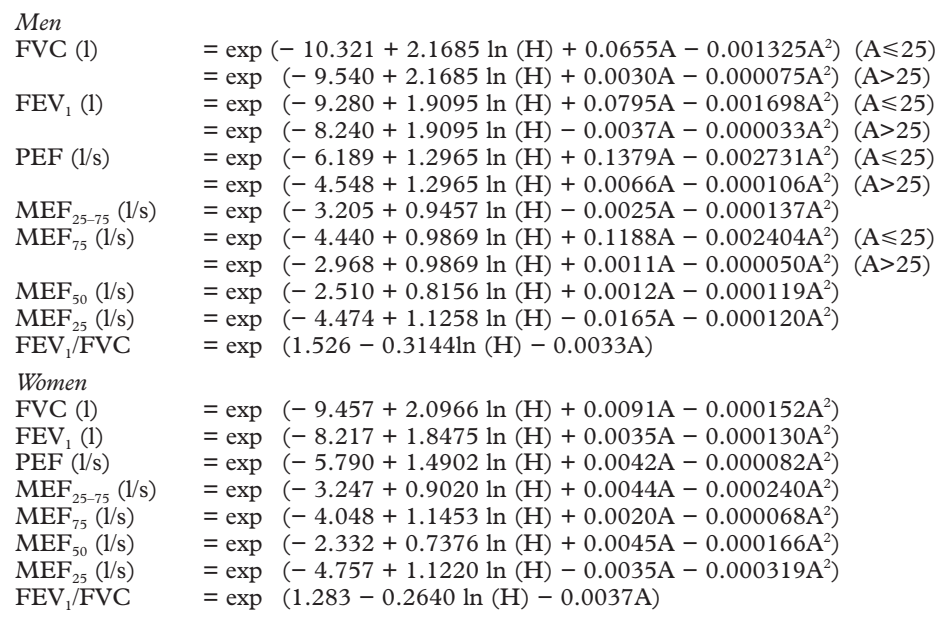

$\mathrm{A}=$ age (years); $\mathrm{H}=$ height $(\mathrm{cm}) ; \exp (x)=\mathrm{e}^{x}$

For a man of 20 years and $180 \mathrm{~cm}$ the predicted value of $\mathrm{FEV}_{1}$ is computed as follows:

$\mathrm{FEV}_{1}=\mathrm{e}^{(-9.280+1.9095 * \operatorname{In}(180)+0.0795 * 20-0.001698 * 400)}=\mathrm{e}^{1.547}=4.696$

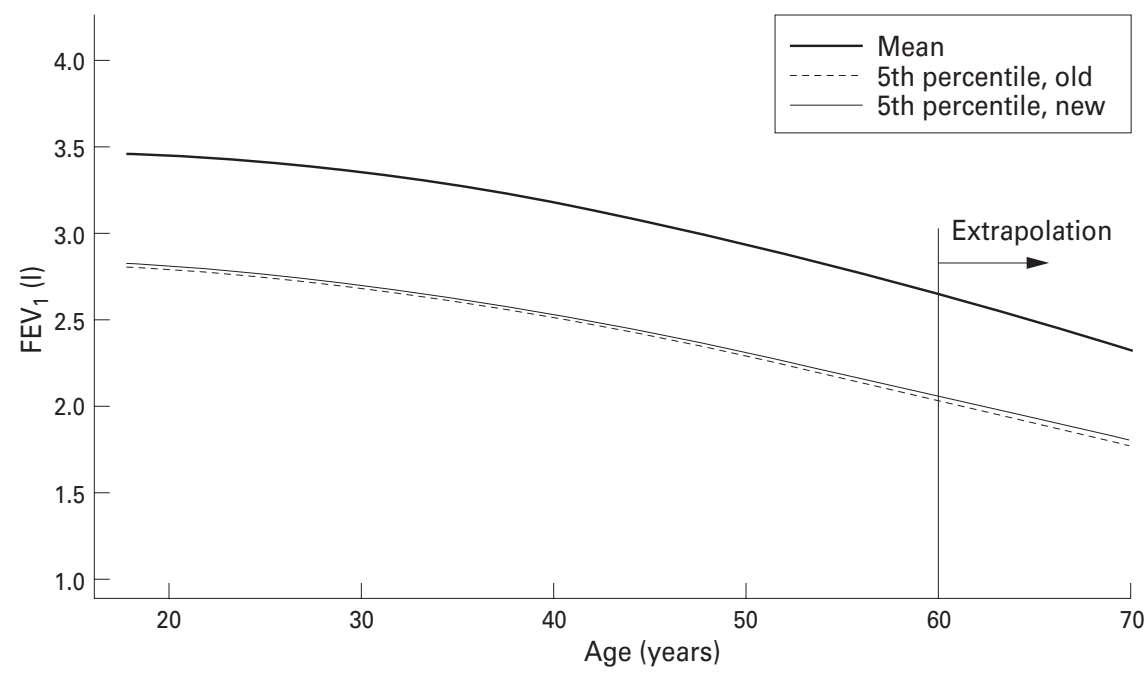

Figure 2 Mean and 5th percentile of FEV, in women of height $1.65 \mathrm{~m}$ as a function of age (based on cross sectional lung function data from healthy never smoking women aged 18-60 years). Original and revised estimates of the 5 th percentiles are shown.

first defined an indicator variable $\mathrm{U}$ taking the value of 1 for residuals $r \leqslant \alpha+\beta$ age and the value 0 for residuals $r>\alpha+\beta$ age and then computed a logistic regression model for $\mathrm{U}$ in terms of the covariates age and age. ${ }^{2}$

The quadratic age term did not reach statistical significance for any of the lung function parameters considered, thus suggesting that the new model was sufficient throughout. In an analogous way we couldverify that the 5 th percentile of $r$ did not significantly depend on the height of the person. Our new estimates of the parameters $\alpha$ and $\beta$ are shown in table 1 . For the sake of completeness we reproduce in table 2 the equations for the means of lung function parameters previously published.
In contrast to our original estimates of the 5 th percentile equations, those derived with the new method appear to provide plausibleextrapolations beyond the age of 60 years for all lung function parameters considered. However, this does not prove the validity of these extrapolations. Only empirical data on lung function from a sample of healthy never smoking subjects older than 60 years could provide truly reliable estimates of lung function parameters at this age. The original and revised estimates of the 5 th percentiles of $\mathrm{FEV}_{1}$ for men and women are shown in figs 1 and 2 .

O BRÄNDLI C SCHINDLER PH LEUENBERGER X BAUR P DEGENS

N KÜNZLI R KELLER A P PERRUCHOUD Zürcher Höhenklinik Wald, 8639 Faltigberg-Wald, Switzerland

1 Brändli O, Schindler Ch, Künzli N, et al and the SAPALDIA team. Lung function in healthy never smoking adults: reference values and lower limits of normal of a Swiss population. Thorax 1996;51:277-83.

2 Baur X, Isringhausen-Bley S, Degens P. Comparison of lung-function reference values. Int Arch Occup Environ Health 1999;72:69-83.

3 Goldstein H, Pan H. Percentile smoothing using piecewise polynomials with covariates. Biometpiecewise polynomials
rics 1992;48:1057-68.

4 Koenker R, Basset G. Regression quantiles. Econometrica 1978;46:33-50.

\section{Course on Pulmonary Pathology}

A course on Pulmonary Pathology designed to provide histopathologists and cytopathologists with an opportunity to study diagnostic lung pathology in a comprehensive manner will be held at the Imperial College School of Medicine, National Heart \& Lung Institute, London on 20-23 June 2000. Further details and application forms are available from Professor B Corrin, Brompton Hospital, London SW3 6NP. Fax: +44 207351 8293. Email: b.corrin@ic.ac.uk. 